\title{
Jungfrau in Gefahr oder mutige Kämpferin? Gender im japanischen Videospiel
}

\section{Marvin Udzik}

\section{Einleitung: Videospiele und Geschlechterrollen}

Diese Arbeit verfolgt das Ziel, die mediale Widerspiegelung des in der japanischen Gesellschaft wachsenden Bewusstseins für Differenzen darzustellen und auf ihre Bedeutung hin zu untersuchen. Der Schwerpunkt liegt dabei auf der Repräsentation von Geschlecht und damit der Wahrnehmung geschlechtlicher Differenz. Diese Entwicklung verfolge ich vor dem Hintergrund der zunehmenden Globalisierung sowie den damit einhergehenden Reaktionen - Öffnung auf der einen, Renationalisierung auf der anderen Seite - und werde in meiner Analyse diesen Linien folgen, d. h. die Reproduktion traditioneller Rollenbilder im Videospiel einerseits und das Verschwimmen der geschlechtlichen Differenzen andererseits thematisieren.

Der Grund dafür, dass ich mich zur Untersuchung solcher Repräsentationen innerhalb der Populärkultur für das Medium Videospiel entschieden habe, ist der große Anklang, den solche Spiele in der Bevölkerung finden. Wie aus Abb.1 hervorgeht, wurden von den beiden japanischen „Videospielgiganten“ Nintendo und Sony allein in der ersten Hälfte des Jahres 2008 insgesamt weit mehr als sechs Millionen Konsolen- und Handheld-Verkäufe erzielt.

Auch was die Verkaufszahlen für Software angeht, haben Nintendo und Sony die Nase ganz vorn: Laut den „Japan Game Charts“ wurden von den erfolgreichsten Nintendo-DS-Spielen bisher über fünfeinhalb Millionen Exemplare verkauft; andere Konsolen können Verkaufszahlen von knapp über drei Millionen (Nintendo Wii) bis hin zu fast vier Millionen Exemplaren (Sony Playstation 2) der beliebtesten Spiele für sich verbuchen (vgl. Japan Game Charts).

Da das Medium Videospiel hohe Verkaufszahlen aufweist, ist davon auszugehen, dass eine breite Masse der japanischen Gesellschaft von diesem populärkulturellen Produkt erreicht wird. Des Weiteren werden Videospiele im Vergleich zu anderen beliebten Produkten japanischer Populärkultur wie Manga, 


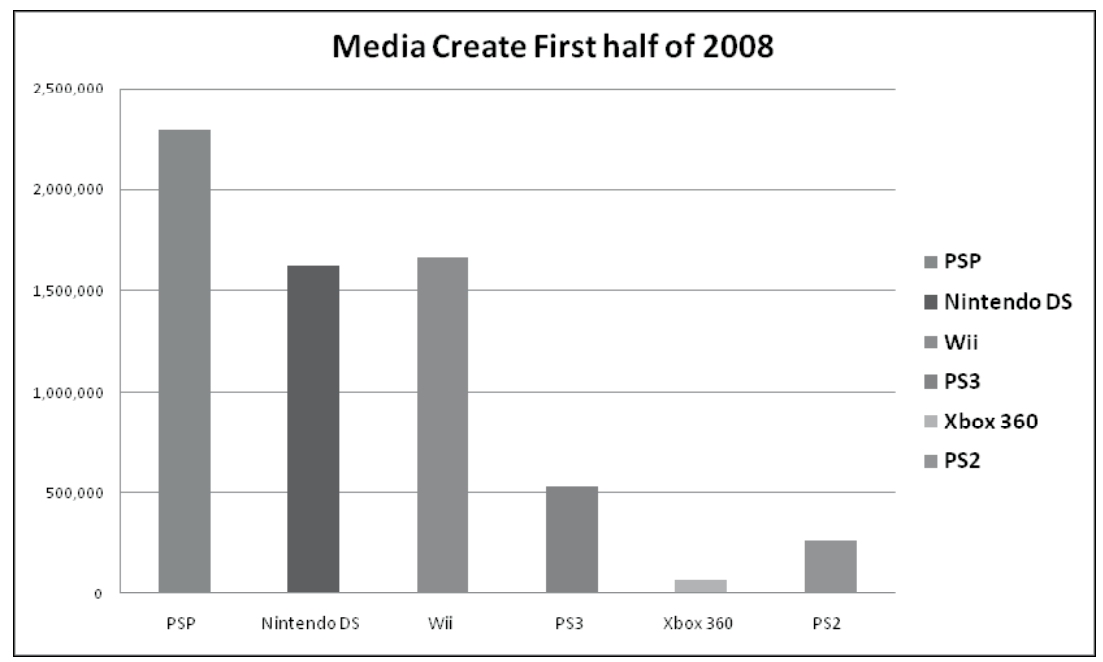

Abbildung 1: Hardware-Verkaufszahlen aus Japan vom 31.12.2007 bis zum 6.7.2008 (Quelle: Media Create)

Anime oder dorama nicht nur passiv konsumiert, sondern fordern Interaktion vom Benutzer (wie es auch analoge Spiele tun). Die Wahrscheinlichkeit einer Beeinflussung des Konsumenten durch das Produkt erscheint mir daher im Bereich des Videospiels höher als bei anderen Produkten, was solche Spiele zu einem interessanten Untersuchungsgegenstand in Bezug auf die Darstellung von (Gender-)Stereotypen und Differenzen macht. Für den weiteren Verlauf der Arbeit ist es daher sinnvoll, sich an zahlreich verkauften und oft gespielten Videospielen der beiden großen japanischen Konzerne Nintendo und Sony zu orientieren, um untersuchen zu können, auf welche Weise und vor allem zu welchem Zweck Charaktere in ihren Spielen Gender-Stereotypen verkörpern oder eben die gängigen Klischees hinter sich und damit Grenzen verschwimmen lassen.

Um dies tun zu können, bedarf es aber natürlich zuerst einmal einer Antwort auf die Frage, wie denn überhaupt die Vorstellungen von einem typischen Mann beziehungsweise einer typischen Frau innerhalb der japanischen Gesellschaft aussehen. Welche Attribute würde man ihnen zuordnen? Welche Eigen- 
schaften werden für welches Geschlecht als wünschenswert angesehen? Zur Beantwortung dieser Fragen und zur Schaffung einer kontemporären Geschlechter-Typologie Japans, von der diese Arbeit ausgehen soll, beziehe ich mich auf die Untersuchung zur Entwicklung der Gender-Rollen in der japanischen Kultur von Sugihara Yoko und Katsurada Emiko (2002). In dieser wurden über mehrere Etappen hinweg unterschiedlich große Gruppen japanischer Männer und Frauen im Alter zwischen 18 und 78 Jahren gebeten, aus einer Liste von 296 Wörtern und Phrasen solche auszuwählen, die ihnen als wünschenswert für einen japanischen Mann beziehungsweise eine japanische Frau erschienen. Dabei ging es nicht darum, welche Eigenschaften die Befragten für sich selbst als wünschenswert erachten, sondern darum, was für die japanische Gesellschaft als wünschenswert erscheint (vgl. Sugihara/Katsurada 2002).

Als für Männer erwünschte Attribute wurden Punkte wie eine starke Persönlichkeit, Führungsqualitäten, ein starker Wille oder Courage ausgewählt, während auf Seiten der Frauen Punkte wie Liebenswürdigkeit, Höflichkeit, Gemütsruhe, die Liebe zu Kindern oder die Empfänglichkeit für die Nöte Anderer genannt wurden. Die Ergebnisse dieser Untersuchung scheinen eine eindeutige Sprache zu sprechen: Der japanische Mann soll verlässlich und widerstandsfähig sein, die japanische Frau familiär, einfühlsam und ruhig. Allein die von mir oben genannten Eigenschaften weisen sehr deutlich bekannte Dichotomien wie rational - emotional, aktiv - passiv, soto (Außenwelt) - uchi (Inneres, eigenes Heim) auf.

Es versteht sich dabei von selbst, dass die Ergebnisse dieser Untersuchung nicht unbedingt die gesellschaftliche Realität widerspiegeln. So liefert beispielsweise eine nachfolgende Untersuchung von Sugihara und Katsurada, in der sie die teilnehmenden Frauen und Männer darum baten, zu beurteilen, inwieweit die eigene Persönlichkeit der herausgestellten weiblichen oder männlichen Gender-Rolle entspreche, das Ergebnis: ,about one third of men and women possessed gender typed personalities whereas two thirds of them had non-gendertyped personalities" (Sugihara/Katsurada 2002). Umso interessanter erscheint es daher zu untersuchen, wie und warum sich in den virtuellen Welten des japanischen Videospiels solche nicht unbedingt realitätsgetreuen gesellschaftlichen 
Idealrollen widerspiegeln oder eben auflösen. Wie werden durch Verwendung dieses populärkulturellen Produktes - sowohl intentioniert als auch unbeabsichtigt - Vorstellungen von entsprechenden Stereotypen oder geschlechtlichen Differenzen vermittelt?

Im weiteren Verlauf meiner Arbeit soll unter Anwendung der oben genannten Ergebnisse als Ausgangspunkt für das typisch Weibliche und typisch Männliche zunächst die Typologie der Geschlechterrollen im Videospiel herausgearbeitet werden; obwohl der Schwerpunkt des Projekts auf Spielen ab dem Jahr 2000 liegt, sollen dabei auch kurz frühere Videospielwerke, die inhaltlich zum Thema beitragen können, Erwähnung finden. Im zweiten Teil der Arbeit sollen solche Charaktere herausgestellt werden, die nicht im eigentlichen Sinne den existierenden Stereotypen zuzuordnen sind, sondern beispielsweise die Rolle des anderen Geschlechtes übernommen haben oder komplett rollenüberschreitend handeln. Bedingt durch das von mir verwendete Material an Videospielen, das ich abhängig von Herausgeber und Erscheinungsjahr des Spiels und vor dem Hintergrund, möglichst mehrere Genres anschneiden zu können, ausgewählt habe, liegt vor allem im zweiten Teil der Arbeit ein gewisser Schwerpunkt auf der Rolle der Frau, während Aspekte zur Rolle des Mannes häufig nur zu Zwecken des Kontrastes herangezogen werden. Im Schlussteil der Arbeit schließlich sollen die erhaltenen Informationen unter Berücksichtigung des gesellschaftlichen Kontextes im Bereich der Videospiel- und Werbeindustrie zusammengetragen und mit einem Ausblick auf mögliche Entwicklungen in der Zukunft ausgewertet werden.

\section{Typologie der virtuellen Geschlechter}

\subsection{Magie und Weiblichkeit}

Für eine nähere Untersuchung der Gender-Typologie im japanischen Videospiel ist es unerlässlich, auch einen Blick auf die früheren Werke der Videospielgeschichte zu richten, da einige der modernen Darstellungen im Videospiel bereits auf eine gewisse Tradition zurückgeführt werden können. Innerhalb meines thematischen Rahmens erscheint mir dazu vor allem das Spiel Lufia II - Rise of the 
Sinistrals $^{1}$ (jap. Estpolis denki II エストポリス伝記 II), ein 1995 auf dem Super Nintendo Entertainment System (SNES) erschienenes $R P G^{2}$, als Beispiel geeignet.

Die Geschichte von Lufia dreht sich um den Helden Maxim, einen Schwertkämpfer mit übernatürlicher Begabung, der dazu bestimmt ist, das Böse in Form der vier Sinistrals zu vernichten. Im Verlauf der Handlung trifft er auf andere Kriegerinnen und Krieger, die ebenfalls dazu auserwählt sind, mit ihm zusammen zu kämpfen; zu der Generalin Selan (jap. Serena) entwickelt Maxim sogar eine Liebesbeziehung, aus der auch ein Kind hervorgeht. Im finalen Kampf gegen die Widersacher muss das Paar schließlich sein Leben lassen, um dadurch den Untergang der Welt verhindern zu können.

Besonders interessant ist dabei die Entwicklung von Selan und ihrer Umwelt. In ihrer ersten Szene im Schloss ihrer Heimatstadt Parcelyte ist sie beim Training mit den anderen (ausschließlich männlichen) Soldaten zu sehen, die sie allesamt mit Leichtigkeit im Kampf besiegt. Auch in den nachfolgenden Szenen erscheint sie kampferprobt, mutig und selbstbewusst, bleibt dabei aber zunächst misstrauisch Maxim und den anderen Charakteren gegenüber. Auf Maxims Frage hin, wie er denn ihr Vertrauen gewinnen könne, gibt ihm Selan zu verstehen: "Strength is not the answer, I can tell you that. ${ }^{\text {" }}$

Dass ihr Charakter neben der starken, selbstbewussten Kämpferin weitere Facetten aufweist, zeigt auch eine andere Szene des Spiels, in der Selan zwei Spitz-

1 Im Folgenden beziehe ich mich auf Lufia, SNES, PAL-Version, Neverland/Nintendo 1996. Hierbei handelt es sich um die europäische Version des Spiels, die - da der erste Teil in Europa nie veröffentlicht wurde - keine 2 in Namen trägt. Chronologisch ist das Spiel übrigens ohnehin vor dem ersten Teil der Serie angesiedelt.

2 RPG (Role-Playing Game, dt. Rollenspiel) bezeichnet ein von der Grundidee her auf Penఓ Rollenspielen basierendes Videospiel-Genre, in dem der Spieler einen Trupp mehrerer Helden steuert und in meist rundenbasierten Kämpfen Erfahrungspunkte gewinnen kann, um mit diesen die Attribute seiner Charaktere (abhängig vom Spiel mit unterschiedlich stark ausgeprägtem eigenem Handlungsfreiraum) wie Stärke, Resistenz, Intelligenz etc. zu verbessern. Inhaltlich folgen die meisten RPGs einer Haupthandlung (innerhalb derer sich der Hauptcharakter meist als auf die ein oder andere Art und Weise ,auserwählt“ entpuppt), während mit Fortschreiten dieser Handlung immer wieder so genannte Sidequests (dt. Nebenhandlungen, ebenfalls von unterschiedlicher Größe und Dauer) zu bewältigen sind.

3 Für dem Spiel wörtlich entnommene Zitate verwende ich hier und im Folgenden eine digital einsehbare Mitschrift (http://forfeit.electromaz.com/lufia2/script/). 
buben verjagt, die ein kleines Mädchen ärgern. Sie tröstet das weinende Kind mit den Worten: „It's not right for them to bully you, but girls shouldn't cry so easily either. [...] A woman's tears ... mean a lot more than a guy's." Beide Zitate lassen vermuten, dass ihr kämpferisches Erscheinungsbild viel mehr einen Schutz darstellt, um mit den Männern konkurrieren und sie so daran hindern zu können, ihre Gefühle zu verletzen. Dabei scheint sie die Emotionalität als den Frauen vorbehalten zu empfinden und sieht sie gleichzeitig als wichtiger an als bloße Stärke (diese Charakterzüge werden auch von ihren spieltechnischen Attributen unterstützt, die weiter unten noch Erwähnung finden).

Bis zu diesem Punkt erscheint Selan also noch als eigenständiger und komplexer Charakter, der um mehr als eine einzige Idee herum entwickelt ist und dessen Wesen von verschiedenen Eigenschaften bestimmt wird. Dies ändert sich jedoch mit der Liebesbeziehung zu Maxim. Etwa in der Mitte des Spiels heiraten die beiden und in wenigen Szenen, die nun auf dem Bildschirm abgespielt werden, vergeht im Spiel ein ganzes Jahr. Schon in der letzten Szene dieses ersten Ehejahres wird anhand eines Dialogs zwischen den Ehepartnern deutlich, dass Selan zumindest auf emotionaler Ebene ihre Unabhängigkeit eingebüßt hat:

Selan: Hey ... Do you love me?

Maxim [...]: Do I have to tell you?

Selan [turns away]: Yes.

Maxim: So you're going to make me say it.

Selan: I need to hear it sometimes ...

Kurz darauf wird schließlich der Sohn der beiden geboren und auch prompt von einem Handlanger des Bösen entführt. Bei der folgenden Rettungsaktion wird Selan aus Sorge um ihr Kind erstmals unachtsam und bringt beide in Gefahr. Auch in den Kämpfen erweist sich Selan nun als weniger nützlich als zuvor: War sie anfangs aufgrund ihrer relativ guten Balance zwischen physischer Stärke und magischer Angriffskraft eine der besseren Figuren, wird sie nun in beiden Attributen von anderen Charakteren übertroffen (siehe unten). Ihre Kräfte scheinen buchstäblich zu schwinden, und nach dem finalen Kampf am Ende des Spiels erscheint sie schließlich vollkommen kraftlos („I was hit [and] can barely speak...", etc.). Eine Rückentwicklung des komplexen, sowohl mit männlichen 
als auch mit weiblichen Attributen versehenen Charakters „Selan“ zu einem Stereotyp für Emotionalität und Mütterlichkeit und die scheinbar aus dieser Entwicklung resultierende Schwäche treten hier deutlich hervor.

Dabei scheint Selans Nützlichkeit im Kampf nicht nur von der Handlung abzuhängen, sondern auch durch die Konstellation innerhalb der Gruppe, also in Relation zu den anderen Charakteren, konstituiert zu werden. So befindet sich Selan in den früheren Episoden des Spiels noch zusammen mit Tia, dem einzigen anderen weiblichen Charakter, sowie den Kraftprotzen Guy und Dekar (jap. Haidekka) in einer Gruppe. Bedingt durch die Unterschiede in der Verfügbarkeit der verschiedenen Waffen, Rüstungsteile und Zauber für die einzelnen Charaktere, bilden sich ganz unterschiedlich gewertete Charakterbilder heraus: So verfügen die männlichen Charaktere Guy und Dekar zwar über eine große physische Stärke, sind aber aufgrund zu geringen Intelligenz-Wertes nicht in der Lage, auch nur einen einzigen Zauber einzusetzen. ${ }^{4}$ Dame Tia hingegen kennt sich mit Zauberei zwar aus, kann dafür im Kampf aber beispielsweise aufgrund mangelnden Abwehr-Wertes nicht sehr vielen gegnerischen Angriffen standhalten. In dieser Konstellation nimmt Selan eine vorrangige Position ein, da sie einerseits als Frau über eine sehr große Auswahl von Zaubern verfügt, die sie im Kampf dank hoher Intelligenz effektiv einsetzen kann, andererseits im Vergleich zu Mitkämpferin Tia - wenn auch nicht im Vergleich zu den männlichen Charakteren - über recht hohe Werte für Stärke und Widerstandsfähigkeit verfügt.

Anders erscheint Selan in der Konstellation der Charaktere nach der Hochzeitsepisode, also etwa ab der zweiten Hälfte des Spiels. Das liegt neben den immer stärker werdenden Waffen und Rüstungen für Guy, die seinen Nachteil, keine Magie verwenden zu können, größtenteils ausgleichen, vor allem an dem Elfen Arty, einem späten Neuzugang in der Gruppe. Als einziger nichtmenschlicher Charakter vereint Arty die Fähigkeiten der anderen Figuren in sich: So kann er in Sachen Stärke beinahe mit den männlichen Charakteren mithalten; außerdem verfügt er über ein noch höheres Arsenal an Zaubersprüchen als die weibli-

4 Ausgenommen ist bei dieser Aufstellung der Hauptcharakter, da dieser in fast allen RPGs - so auch hier - als „Held“ standardmäßig in vielen bis allen Bereichen überdurchschnittlich hohe Werte aufweist. 


\section{Marvin Udzik}

chen Charaktere. Der Unterschied zu Selan wirkt in dieser Zusammenstellung ähnlich wie der Unterschied zwischen Selan und Tia zuvor, nur dass dieses Mal Selan auf der schlechteren Position steht. Auch im Bereich der Kampftauglichkeit lässt sich also, bedingt durch die Umstände der voranschreitenden Handlung des Spiels, eine Art Rückentwicklung feststellen. An die Stelle des ambivalenten Charakters mit (videospieltypischen) Eigenschaften beider Geschlechter ist nun Arty getreten - als Elf ein nicht nur sehr androgynes, sondern in den Dialogen des Spiels auch wiederholt als den Menschen überlegen dargestelltes Wesen („I live longer than humans and sense certain patterns of energy that humans cannot", etc.); ihm lassen sich weder von seinen Statuswerten her noch auf handlungsbezogener Ebene typisch männliche oder typisch weibliche Attribute zuordnen. ${ }^{5}$

Was aber sollen uns diese Entwicklungen nun sagen? Lautet die Aussage hinter all dem, dass es nur übermenschlichen Wesen möglich ist, ein wirkliches Ausbrechen aus den bestehenden Geschlechterrollen zu vollziehen? Und lag es in der Absicht der Entwickler, diesen Umstand als gut zu schildern? Oder soll die Rückentwicklung Selans vielleicht als Strafe betrachtet werden, gewissermaßen als ein Aufruf für neue Geschlechterbilder?

Dies scheint mir nicht eindeutig zu beantworten. Sicher ist jedoch, dass klassische Werteverteilungen wie bei Lufia - Kraft und Widerstandsfähigkeit bei den männlichen, Intelligenz und Zaubervermögen bei den weiblichen Charakteren - absolut keine Seltenheit darstellen, sondern im RPG seit der Entstehung des Genres existieren. Im 1994 erschienenen sechsten Teil der wohl berühmtesten japanischen RPG-Serie Final Fantasy ${ }^{6}$ beispielsweise, können Zauber nicht wie bei Lufia einfach käuflich erworben werden, sondern sind erst durch die Verbindung mit bestimmten gottähnlichen Wesen zu erlernen. Die einzigen beiden

5 Obwohl Arty vom Spiel selbst als „männlicher“"Elf vorgestellt wird, scheint es seit jeher Verwirrungen und Uneinigkeiten über das Geschlecht dieses Charakters zu geben. Dies mag unter anderem daran liegen, dass "Arty“ in der amerikanischen Version des Spiels seinen Namen nur noch als Spitznamen trägt, aber eigentlich „Artea“ heißen soll. Dies beeinflusste auch die europäischen Versionen; so wurde beispielsweise in der deutschen Version des Spiels der Name „Artea“ komplett beibehalten.

6 Ich beziehe mich auf Final Fantasy VI, Sony Playstation, PAL-Version, Square 2002. Es handelt sich hierbei um eine Neuauflage des ursprünglich für das SNES veröffentlichten Spiels. 
Charaktere, die dabei eine Ausnahme bilden und aus sich selbst heraus die Fähigkeit zur Zauberei entwickeln, sind gleichzeitig auch die einzigen beiden Frauen. Es scheint so, als ob Frauen nicht mehr nur durch die ihnen üblicherweise zugeordnete Emotionalität punkten können, sondern durch Verwendung der Magie zu einer Art Spiritualität gelangen, die ihnen nun jenseits von physischer Stärke einen Raum zum Wirken gibt.

\subsection{Damsel in Distress}

Neben den magischen Frauen der vielfältigen $R P G$-Welten existieren aber auch solche Bilder, die weibliche Charaktere nahezu nutzlos oder sogar als störend erscheinen lassen. Ein häufig anzutreffendes Schema ist dabei das der Damsel in Distress (dt. etwa "Jungfrau in Gefahr“). Ursprünglich aus dem Mittelalter kommend und als Bezeichnung für Frauen in einer Notlage (vor allem durch Scheidung) verwendet (vgl. Forschungsinstitut für Mittelalter und Renaissance 1985: 147), ist das Phänomen seit langem in den virtuellen Welten der Videospiele gerade dann anzutreffen, wenn es in der Rahmenhandlung darum geht, in der Rolle eines männlichen Helden dessen geliebte Frau (meist eine Prinzessin, ähnlich den mittelalterlichen Rittergeschichten) zu erretten.

Dass sich dieses Prinzip oft über ganze Serien hinweg erstreckt und dabei nahezu unverändert bleibt, zeigt beispielsweise die Super-Mario-Serie des Entwicklers Nintendo. Die einzelnen Teile dieses Jump'n'Run $n^{7}$-Meilensteins lassen sich nämlich in ihrer Handlung in nur einem Satz beschreiben: Die Angebetete von Klempner Mario wird entführt und muss von ihm aus den Klauen eines bösen Widersachers gerettet werden - die Rettung selbst stellt dabei auch schon das Ziel des Spiels dar. Interessant ist, dass sich innerhalb der aus mittlerweile drei-

7 Das Genre Jump'n'Run (dt. „Springen und Rennen“) umfasst - wie der Name schon sagt - solche Videospiele, bei denen sich die Spielfigur laufend und springend fortbewegt. Im Gegensatz zum $R P G$ ist dieses Genre also weniger auf Strategie, sondern eher auf Geschicklichkeit aus, weshalb das Bestehen der einzelnen Levels (Ebenen, die der Spieler durchschreiten muss) auch meist vor dem Erzählen einer Geschichte in den Fokus des Spiels rückt. Obwohl der Name des Genres aus dem Englischen stammt, ist er dort eher selten gebraucht; stattdessen findet der Begriff Platform Game oder einfach Platformer häufig Verwendung. 


\section{Marvin Udzik}

zehn Hauptteilen ${ }^{8}$ bestehenden Serie (seit $1981^{9}$ ) zwar das Damsel in DistressPrinzip gehalten hat, dabei aber die Rollen der zu errettenden Frau und des Bösewichts, der sie entführt, über die Zeit ausgetauscht wurden. So wird noch im allerersten Videospiel der Mario-Serie, Donkey Kong, Marios Freundin Pauline von dem Riesenaffen Donkey Kong gekidnappt; dies bleibt auch ihr einziger Auftritt.

In dem ebenfalls noch auf dem Game Boy erschienenen Super Mario Land schließlich ist es Prinzessin Daisy, die von dem übermächtigen Weltraummonster Tatanga entführt und als Geisel genommen wird. Obwohl damit auch ihr Auftritt innerhalb der Hauptserie abgeschlossen ist, hat Daisy immerhin nachfolgende Auftritte in diversen Sport-, Renn- und Kampfspielen, die als Spin-offs der Serie entstanden (z. B. Super Mario Kart, Super Mario Tennis, Super Smash Bros.). Als Damsel in Distress durchgesetzt hat sich schließlich aber die schon optisch klischeebeladene Prinzessin Peach ${ }^{10}$, die durch ihr Talent, immer wieder in die Klauen von Echsenkönig Bowser (jap. Kuppa) zu geraten, sowie durch die scheinbar vollkommene Unfähigkeit, sich in irgendeiner Weise selbst aus der Klemme zu helfen, Mario überhaupt erst den Sinn seines virtuellen Lebens verleiht. Dass sich dabei über die Jahre hinweg weder das pinkfarbene Prinzessinnenkleid noch ihre Rolle als passive Maid in Nöten großartig weiterentwickelt hat, versteht sich dabei fast schon von selbst. ${ }^{11}$

Es ist natürlich klar, dass die Peach zu Grunde liegenden stereotypen Attribute einer Frau in Not, die von der Fähigkeit und dem Mut ihres Mannes abhängig ist,

8 Zu den Hauptteilen werden ausschließlich die Jump'n'Run-Teile der Serie gezählt, d. h. nicht einbezogen sind nachfolgende Spin-offs in anderen Genres.

9 Die Serie an sich existiert in Form von Arcade-Spielen, sprich: an Automaten in Spielhallen, schon seit 1981. Im Bereich der Heimkonsolen hatte sie 1985 mit Super Mario Bros. für das Nintendo Entertainment System (NES) ihr Debüt.

${ }^{10}$ Außerhalb Japans war Peach bis 1996/97 (mit dem Erscheinen von Super Mario 64) unter dem Namen Toadstool bekannt (vgl. http://en.wikipedia.org/wiki/Princess_Peach).

${ }^{11} \mathrm{Zu}$ bemerken ist hier, dass sich dieses Prinzip nicht auf das komplette Mario-Universum anwenden lässt. So lässt sich Peach beispielsweise in Super Mario RPG: Legend of the Seven Stars, einem RPG-Spin-off der Serie, als spielbarer Charakter in die Gruppe wählen und kann so aktiv am Spielgeschehen teilnehmen. Auftritte dieser Art sind allerdings erstens selten und gehen zweitens immer mit der für Peach typischen Anbetung Marios als ihren Helden einher. Dies zeigt sich in Super Mario RPG beispielsweise an Aussagen wie „Mario, you’re my knight in shining armor!“ 
unweigerlich an ihre Rolle als Damsel in Distress gebunden (und gewissermaßen ein Teil von ihr) sind. Dass sich das Klischeebild um die pinke Prinzessin aber auch dann nicht ändert, wenn sie mit ihrem geliebten Mario die Rollen tauscht, lässt sich seit dem Erscheinen ihres ersten eigenen Videospiels, Super Princess Peach, im Jahr 2006 erkennen, in dem sie erstmalig die Rolle der Protagonistin übernimmt und als solche diesmal Mario aus der Gefangenschaft zu befreien versucht.

Von der Grundidee her also eigentlich innovativ in Bezug auf die bisher gebildeten Gender-Rollen, bedient sich dieses Spiel dennoch der stereotypen Dichotomie „emotionale Frau versus rationaler Mann“, ohne dabei wirklich die existierenden Geschlechterbilder zu durchdringen. Stehen Mario in seinen eigenen Abenteuern (je durch Einsatz eines bestimmten Objektes) Fähigkeiten wie das Schießen von Feuerbällen, das Werfen von Hämmern oder sogar temporäre Unverwundbarkeit zur Verfügung, muss sich Peach bei ihrer Rettungsaktion anscheinend ganz auf die Waffen einer Frau verlassen: ihre Emotionen. So ist es dem Spieler durch Einsatz des im Nintendo DS eingebauten Touchscreens möglich, auf Knopfdruck diverse Gefühle in der Prinzessin hervorzurufen. Dabei fällt die Wahl vor allem zwischen Freude (jap. 喜), die Peach in die Lüfte schweben lässt, Zorn (jap. 怒), der sie in eine wandelnde Flamme und damit eine gefährliche Waffe verwandelt, und Trauer (jap. 哀), die sie ganze Wellen von Tränen weinen lässt, um so diverse Pflanzen zum Wachsen oder Flammen zum Erlischen zu bringen. ${ }^{12}$ Um den Nachschub an künstlich erzeugbaren Gemütsschwankungen zu gewährleisten, muss Peach im Laufe ihrer Mission immer wieder diverse Kristalle aufsammeln, die ihren Vorrat an emotionaler Energie wieder aufladen. Sollte gerade kein Schmuck in der Nähe sein, steht der Prinzessin als Waffe noch immer ihr gelber Schirm zur Verfügung - der jedoch in erster Linie zum sanften Gleiten durch die Lüfte gedacht ist.

Mit Blick auf Super Princess Peach lässt sich also nicht verleugnen, dass kontinuierlich gewisse Gender-Stereotypen bedient werden. Gonzalo Frasca, selbst

${ }^{12}$ Als vierte Emotion existiert weiterhin „Behaglichkeit“ (jap. 楽, in der dt. Version „Sanftmut“), die allerdings lediglich die Lebensenergie von Peach regeneriert und keinen wirklichen Gefühlsausbruch herbeiruft. 
Akademiker und Videospielentwickler, meint dazu: „What is shocking is that from all the possible design options available, the creators of this game had to frame the princess as an emotionally unstable person" (Ransom-Wiley 2006). Eine Entwicklung in diesen über Jahre aufgebauten Geschlechterrollen scheint dabei nicht zu erwarten zu sein.

Dass aber auch Weiterentwicklungen eines Charakters innerhalb der Rolle als Damsel in Distress möglich sind, zeigt uns beispielsweise die Videospiel-Serie The Legend of Zelda (jap. Zeruda no densetsu ゼルダの伝説). In dieser seit 1986 bestehenden vierzehnteiligen Adventure ${ }^{13}$-Serie ist es die namensgebende Prinzessin Zelda, die sich immer wieder entführen lässt, nur um anschließend vom tapferen Schwertschwinger Link gerettet zu werden. Zelda erweist sich jedoch als wesentlich nützlichere Spielfigur als Prinzessin Peach. Teil der vorgegebenen Handlung ist zum Beispiel, dass Zelda, bevor sie von den Klauen des Bösen ergriffen wird, noch schnell das sogenannte Triforce, ein innerhalb der Geschichte des Spiels äußerst mächtiges Artefakt, zerstört und die Einzelteile in alle Winde zerstreut.

In Ocarina of Time (jap. Toki no okarina 時のオカリナ) und The Wind Waker (jap. Kaze no takuto 風のタクト) greift Zelda darüber hinaus auch aktiv ins Spielgeschehen ein. So unterstützt sie Link bei Ocarina of Time im letzten Gefecht gegen den Erzrivalen Ganon, indem sie - durch den Einsatz von Magie - diesen an der Fortbewegung hindert, so dass Link mit seinem Schwert das Übrige tun kann. Anschließend sorgt Zelda (unter Mithilfe anderer Magier) dafür, dass der Bösewicht endgültig verbannt wird. In The Wind Waker schließlich tritt Zelda in Form der Piratenbraut Tetra auf, die Links Gegner während des Finalkampfes mit magischen Lichtpfeilen beschießt.

Dass im Vergleich zur Super Mario-Serie in The Legend of Zelda mit dem Klischee der Jungfrau in Not zumindest in Teilen gebrochen wird, zeigt sich einerseits an der aktiveren Rolle der Frau (Zelda trägt zur Handlung bei und

${ }^{13}$ Das Videospiel-Genre Adventure ist, ähnlich dem RPG, sehr auf Inhalt und Story fokussiert, weist dabei aber meist nur einen spielbaren Charakter auf und verläuft nicht in rundenbasierten (strategischen), sondern in direkten Kämpfen, bei denen Geschicklichkeit und Können eine Rolle spielen. Daher könnte man ein Adventure-Spiel also grob als Hybrid eines RPGs und eines Jump'n'Runs bezeichnen. 
nimmt an Kämpfen teil, Peach tut nichts dergleichen) sowie an dem erneuten Einsatz von Magie, schon in frühen RPGs ein Zeichen von „Nützlichkeit“ vor allem bei weiblichen Charakteren. Erwähnt werden muss jedoch, dass es sich in beiden Fällen in erster Linie um Figuren handelt, die dazu erschaffen wurden, dem männlichen Hauptcharakter den Sinn seines Abenteuers zu geben; unterschiedlich ist dabei lediglich der persönliche Einfluss und die Teilnahme dieser „Zweckfigur“, ohne dass dabei eine wirkliche Gleichberechtigung existieren würde. Mit solchen weiblichen Charakteren, die in Schlüsselpositionen oder gar in der Hauptrolle zum Spielgeschehen beitragen, setzt sich der folgende Teil der Arbeit auseinander.

\section{Grenzüberschreitungen}

\subsection{Eine erfolgreiche Revolution?}

Bevor eine Überwindung der bisher dargestellten virtuellen Gender-Typologie anhand von einzelnen Videospielen beziehungsweise anhand des dort festzustellenden Verschwimmens der typischen Geschlechterrollen am Beispiel einzelner Charaktere untersucht wird, soll hier noch einmal der analytische Rahmen des Projekts durch bisher gewonnene Erkenntnisse ergänzt werden. Eine Tabelle zur Typologie des Weiblichen und Männlichen zeigt die Ergebnisse von Sugiharas und Katsuradas Studie (A) sowie die in dieser Arbeit bisher getroffenen Feststellungen zum Computerspiel (B) (vgl. Abb. 2).

Wie aus der Tabelle ersichtlich, kommt der männliche Charakter in den untersuchten Videospielen dem gesellschaftlichen Wunschtyp eines japanischen Mannes nahezu gleich: Es sind physische Stärke und ein starker Wille ebenso wie Courage und Furchtlosigkeit, die sein heldenhaftes Wesen begründen; es ist daher nicht verwunderlich, dass noch immer im Großteil der existierenden Videospiele die Hauptrolle von einem männlichen Charakter übernommen wird. In Bezug auf die weiblichen Charaktere hingegen scheinen fiktionale Figur und gesellschaftliches Ideal nicht komplett übereinzustimmen: Obwohl auch Punkte wie Naivität oder Unschuld in den Umfragen genannt wurden, scheint das Bild der sanften, emotionalen Frau, das für die Gesellschaft scheinbar als wünschenswert erachtet wird, im Videospiel eher in ein negatives Licht gerückt zu 


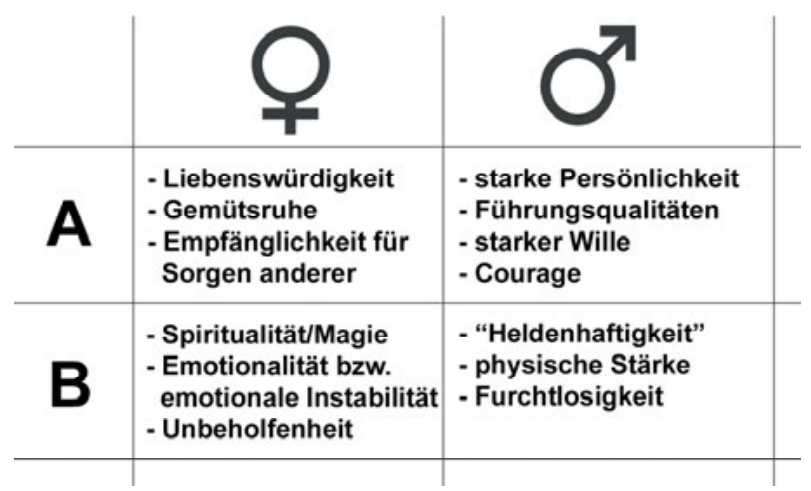

Abbildung 2: Tabelle zur Typologie des Weiblichen und Männlichen.

werden. Zwar wurde auch festgestellt, dass es die virtuellen Frauen in einigen Spielen - vorrangig durch Einsatz von Magie - zu einer gewissen Wichtigkeit und Nützlichkeit als Charakter bringen können, allerdings beschränkt sich diese Eigenschaft zumeist auf die spieltechnische Ebene. Eine wahre Gleichberechtigung müsste sich dabei aber auch auf die charakterliche Ebene ausweiten. ${ }^{14}$

Es ist also wichtig, bei der Analyse ambivalenter Charaktere, die Differenzen zu den geschlechtlichen Stereotypen aufweisen, darauf zu achten, dass sich diese Ambivalenz auch auf beiden Ebenen des Videospiels wiederfinden lässt. Ein weiblicher RPG-Charakter kann trotz hoher Werte für Stärke und Resistenz, die ihm spieltechnisch eher männliche Attribute verleihen würden, auf der charakterlichen Ebene, d. h. bei der Portraitierung seiner Persönlichkeit, nach wie vor einen femininen Stereotypen darstellen, wenn genannte Attribute vorhanden sind.

So liefert beispielsweise im Anime-RPG Grandia II die Dämonin Millenia zunächst eher untypische Werte: Mit hoher physischer und magischer Angriffskraft sowie ihrem großen Arsenal mächtiger Zaubersprüche erscheint sie, wie

14 „Spieltechnische Ebene“ meint alle Faktoren eines Videospiels, die sich mit der Spieltechnik an sich befassen, d. h. beispielsweise die Statuswerte der einzelnen Charaktere, die Nützlichkeit einer Figur im Kampf, figurenbezogene Spezialfähigkeiten, usw. Die Ebene, die sich mit der Persönlichkeit der Charaktere beschäftigt und damit, was sie innerhalb der Geschichte des Spiels sagen und tun, wird hier als „charakterliche Ebene“ bezeichnet. 
die frühe Selan, ein eher ambivalenter Charakter zu sein. Dass dies nicht über die spieltechnische Ebene hinausgeht, macht jedoch nicht nur ihr Erscheinungsbild deutlich.

Neben der Tatsache, dass sich Millenia gegen Ende zum Guten bekehren lässt, da sie sich zu Ryūdo, dem männlichen Helden des Spiels, hingezogen fühlt, weist sie auch während der gesamten Geschichte immer wieder stereotype Attribute in Form einer gewissen Unbeholfenheit auf. So gelingt es ihr beispielsweise nicht, ihre Zugehörigkeit zum Dämonenkönig Valmar, dem eigentlichen Widersacher des Protagonisten, lange zu verbergen:

Millenia: Serves you right to be toying with the "Wings of Valmar!"

Ryūdo: What was that? Who ... or what ... ARE you!?

Millenia: Oops! I said that out loud, didn't I? Never mind me. ${ }^{15}$

Neben Auftritten dieser Art scheint Millenia auch emotional instabil zu sein, wie zum Beispiel folgendes Gespräch zwischen ihr und Skye, Ryūdos sprechendem Hausvogel, deutlich macht:

Millenia: Aww ... you're a sweetheart, aren't ya? This should be fun! [...]

Skye: This woman is a couple eggs shy of a basket.

Millenia: Hey, BIRD! Just how badly do you want to be turned into charcoal!?

Obwohl aus spieltechnischer Sicht nicht erkennbar, macht Millenias gesamtes Auftreten aus ihr eindeutig einen Stereotypen auf charakterlicher Ebene. Ob und wie sehr die Entwicklung solcher Charaktere für Videospiele dennoch als progressiv zu bewerten ist, bleibt dabei wohl jedem selbst überlassen. Da die Veränderungen aber nicht die Persönlichkeit der Figur betreffen, liefern diese im Prinzip nur einen „Scheinausbruch“ aus den gängigen Konventionen. Es können daher nur solche Figuren als wirklich rollenbrechend bewertet werden, die ambivalente Züge auf beiden Ebenen des Spiels aufweisen.

\subsection{Ambivalenz und neue Geschlechterbilder}

Dazu gehören zunächst auch solche Charaktere, die entweder die Rolle des anderen Geschlechts angenommen haben oder sogar typische Attribute beider Ge-

${ }^{15}$ Für dem Spiel wörtlich entnommene Zitate verwende ich hier und im Folgenden eine digital einsehbare Mitschrift (http://www.gamefaqs.com/console/ps2/file/530934/34789). 


\section{Marvin Udzik}

schlechter aufweisen. Ein gutes Beispiel für Erstere sind Mia und Elk aus dem Videospiel .hack//infection (jap. .hack//感染拡大Vol.1). So erscheint Mia als mutige und draufgängerische Kriegerin mit erstaunlich großer Klinge, die hauptsächlich im Bereich physischer Attribute hohe Werte aufweist. Elk hingegen wird als schüchterner junger Magier dargestellt, der zwar mit Zaubersprüchen gut umzugehen weiß, Gefahren generell aber lieber meidet und eigentlich nur in der Nähe seiner Angebeteten Mia sein möchte, weshalb er bereits früh im Spiel aufgrund ihres Interesses an Hauptcharakter Kite eine Art Eifersucht auf eben diesen entwickelt. Anzumerken ist hier, dass Mia zwar keine menschliche Figur, sondern eine Art Katzenwesen ist, es sich bei .hack//infection allerdings auch um ein simuliertes $M M O R P G^{16}$ handelt, d. h. es werden fiktive Spieler, die wiederum in die Rolle der anzutreffenden Charaktere geschlüpft sind, assoziiert.

Solche Charaktere, denen Attribute beider Geschlechtertypologien innewohnen und die daher in beiden Rollen agieren, gehören im Bereich der Videospiele fast immer auch zu den „unvollkommenen“ ambivalenten Figuren, da sich die Charaktere auf spieltechnischer Ebene innerhalb eines Spiels nur selten aus dem bekannten Muster lösen, sondern sich meistens nur innerhalb dessen entwickeln. Häufig anzutreffen sind hier weibliche Charaktere, die im spieltechnischen Bereich Werte einer typisch männlichen Figur aufweisen (d. h. hohe Werte im Bereich physischer Stärke und Resistenz, eher geringe magische Fähigkeiten und ggf. geringe Werte für Geschwindigkeit), auf der charakterlichen Ebene allerdings ambivalent dargestellt werden. Ein gutes Beispiel hierfür ist der Charakter Lin aus dem RPG Breath of Fire: Dragon Quarter. Sie beweist mit Führungsqualitäten, Mut und einem starken Willen eindeutig typisch männliche Qualitäten, ist aber andererseits auch typisch weiblich: sehr liebevoll und empfänglich für die Sorgen anderer. Interessant ist dabei, dass ihre männlichen Attribute hauptsächlich in Interaktion mit dem Hauptcharakter Ryū zum Vorschein treten, während ihr typisch Weibliches vor allem in Bezug auf Nina, dem dritten und letzten spielbaren Charakter, zur Geltung kommt.

${ }^{16}$ Ein MMORPG (Massively Multiplayer Online Role-Playing Game) gehört eigentlich zu den Computerspielen und beschreibt ein $R P G$, an dem viele Spieler aus aller Welt online teilnehmen und sich in virtuellen Welten treffen können. Mit Beginn eines solchen Spiels geht gewöhnlich die Auswahl oder auch das Entwerfen eines eigenen Charakters einher. 
So findet bereits die erste Begegnung zwischen Lin und Ryū in einem Kampf statt, da beide aus zunächst unterschiedlichen Motiven heraus Nina unter ihren Schutz bringen wollen. Bereits das erste Gespräch zwischen beiden beginnt mit: "You can’t help her. Go, and leave her with me. ${ }^{\text {“17 }}$ Und obwohl Ryū sie von seiner Sichtweise zu überzeugen versucht, erwidert Lin nur: „I don't care. I've been a rebel from the start [...]". Im weiteren Verlauf des Spiels jedoch einigen sich beide und versuchen Nina, die aufgrund einiger in der Vergangenheit an ihr durchgeführter Experimente immer schwächer wird, zu helfen. Auf der beschwerlichen Reise, die sie dabei antreten müssen, kümmert sich Lin nahezu mütterlich um das vom Spiel selbst noch als „Kind“ bezeichnete Mädchen, wie auch an Aussagen wie „Just a little bit further, Nina. Are you okay?“ oder „I'll watch over Nina“ immer wieder deutlich wird.

Interessant ist hier die Tatsache, dass bei Lin - so wie bei allen weiblichen Charakteren ihrer Art - die als ideal weiblich herausgestellten Attribute nicht mehr, wie bei den stereotypen Charakteren, ins Negative verdreht sind, sondern dass diese Charaktere tatsächlich zumindest teilweise Idealeigenschaften eines japanischen Mannes und einer japanischen Frau besitzen. Allein die Tatsache, dass sie spieltechnisch betrachtet meist männlich-stereotyp sind, unterstützt dieses Auftreten nicht; dies scheint mir allerdings hauptsächlich durch die generelle Funktionsweise (und den für den Spieler gegebenen Rahmen innerhalb der verschiedenen Genres) von Videospielen begründet. Im direkten Vergleich zu allen bisher untersuchten Figuren erscheinen Bi-Gender-Charaktere wie Lin die am wenigsten stereotypen zu sein.

Die Suche nach neuen Geschlechterbildern im Videospiel, d. h. nach solchen Figuren, die weder typisch männliche, noch typisch weibliche - einschließlich der ins Negativ verkehrten - Attribute und Eigenschaften besitzen, gestaltet sich da schon schwieriger. Ein Charakter, der ein neues Geschlechterbild aufweisen kann, darf weder mutig und entschlossen, noch liebevoll und aufmerksam oder emotional instabil und unbeholfen sein, und sollte nach Möglichkeit auch auf spieltechnischer Ebene nicht die gängigen Werte-Schemata aufweisen. Da ein

${ }^{17}$ Für dem Spiel wörtlich entnommene Zitate verwende ich hier und im Folgenden eine digital einsehbare Mitschrift (http://www.dragon-tear.net/bofdq-englishscript.txt). 
solches Profil aber generell sehr negativ anmuten und sich in Teilen auch selbst widersprechen würde, kann zunächst einmal gesagt werden, dass in den untersuchten elf Videospielen kein einziger Charakter zu finden ist, der wirklich komplett von den typischen Attributen gelöst auftritt (warum dies so ist, soll weiter unten geklärt werden). Des Weiteren kann gesagt werden, dass bei typischen Protagonisten und auch Antagonisten ein gewisser Mut und ein starker Wille, ein bestimmtes Ziel zu erreichen, eigentlich immer gegeben sind. Charaktere, die zumindest teilweise Aspekte neuer Geschlechterbilder aufweisen, sind daher normalerweise solche Figuren, die nur durch äußere Umstände in ein Abenteuer gezogen werden und daher zunächst keine eigenen Ambitionen haben.

So ergeht es zum Beispiel auch dem Charakter Mitsuru Kirijō in Shin Megami Tensei: Persona 3 aus dem Jahr 2006. Deren Großvater hat vor seinem Tod einer Gruppe von Wissenschaftlern angehört, mit einer dunklen Macht experimentiert und so Unheil über die Welt der Gegenwart gebracht. Dass Mitsuru nun in den Kampf gegen dieses Unheil zieht, betrachtet sie selbst eher als Bürde: „Besides ... some of us were never given a choice. I ... [...] I guess you could say it's my way of atoning for the past. ${ }^{18}$ Darunter leidet ihr Einsatz allerdings nicht, wie sich immer wieder an ihren Bemühungen im Kampf und auch an der Organisation der Missionen zeigt; ihre Ideale und Vorstellungen versucht sie in ihrer Rolle als Schülersprecherin auch an andere zu vermitteln, wie beispielsweise bei ihrer Ansprache zum neuen Schuljahr deutlich wird:

As I begin my term as Student Council President, I'd like to share with you my vision for this coming year. It is my firm belief that each of us must accept the responsibility of bettering our school. However, change cannot occur without sustained effort and an unprecedented level of commitment. That is why we must restructure our daily lives to accommodate this lofty goal. I'd like each of you to dig deeply into your well of motivation, and re-evaluate your convictions ... To imagine a bold new future without losing sight of the realities around you. That is the key. I am certain that many of you have your own visions of the future ... For us to reap the full benefits of our education, your participation, ideas, and enthusiasm are essential. Thank you.

${ }^{18}$ Für dem Spiel wörtlich entnommene Zitate verwende ich hier und im Folgenden eine digital einsehbare Mitschrift (http://www.gamefaqs.com/console/ps2/file/932312/50852). 
Schwieriger scheint es Mitsuru jedoch manchmal zu fallen, zwischenmenschliche Kontakte zu knüpfen. Zwar versteht sie sich mit Mitbewohner Akihiko, mit der mädchenhaften Yukari hingegen kann sie zunächst nicht viel anfangen: „To tell you the truth, you're not the easiest person to relate to." Generell erscheint Mitsuru im Großteil des Spieles zwar freundlich, oft jedoch auch kühl und uninteressiert am Leben der Anderen. Dies gipfelt gegen Ende des Spiels im Tod von Mitsurus Vater, woraufhin diese kurzzeitig jegliche Motivation verliert: „Why are you still concerned about me? Our days of fighting together are over. We have no common goal to fight for, no enemies to defeat ... no reason to be friends." Schließlich ist es aber Yukari, die Mitsuru beisteht und ihr zu neuen Perspektiven verhilft („I'll never look back again”, etc.).

Obwohl Mitsuru also typische Eigenschaften wie Führungsqualitäten und Mut aufweist, kommen mit ihr auch ganz neue Attribute zum Vorschein: Verantwortungsbewusstsein, Vernunft, Fleiß und auch eine gewisse emotionale Distanz. Damit ist ihr zumindest partiell ein Ausbruch aus dem Bekannten gelungen, auch wenn teilweise noch Überschneidungen bestehen. Interessant ist außerdem, dass Mitsuru am Ende des Spiels - nach dem Gespräch mit der recht stereotypen Yukari - emotional offener und aufmerksamer wird, also Attribute des typisch Weiblichen annimmt. Dadurch erscheint Mitsuru schließlich auch wie ein sehr ambivalenter Charakter, der in beiden Rollen agieren kann, aber ohne dabei ihre untypischen Eigenschaften aufzugeben. Mitsuru verkörpert also, wie andere Charaktere ihre Art, immer auch einen Teil des Typischen, welches aber mit dem Ansatz für eine neue Geschlechterrolle verbunden ist und ihn stützt.

\section{Resümee: Gendersynkretismus mit Innovation}

Zusammenfassend kann gesagt werden, dass in den untersuchten Videospielen hauptsächlich zwei verschiedene Typen von weiblichen Charakteren auftauchen: Auf der einen Seite die liebevolle, emotionale Frau, die von der Liebe und Fürsorge eines Mannes abhängt und auf dessen Mut baut, dabei ggf. auch selbst unterstützend mitwirkt (z. B. die späte Selan, Peach, Zelda), auf der anderen Seite die starke und selbständige Frau, die in ihrer Gender-Rolle ambivalent auftreten und teilweise Aspekte neuer Geschlechterbilder umschließen kann - ohne aber 
dabei ein vollkommen neues Bild zu verkörpern. Interessant daran ist, dass erstere schon seit dem Ursprung moderner Videospiele in den 1980ern auftauchen, letztere hingegen erst seit dem Ende der 1990er beziehungsweise mit Beginn des neuen Jahrtausends vorzufinden sind.

In dieser Entwicklung spiegelt sich auch ein Wandel in den Ansichten der Menschen innerhalb der japanischen Gesellschaft wider, der sich auch in einer Untersuchung des Ministry of Foreign Affairs of Japan (MOFA) zeigt. Laut dieser Studie ist der Prozentsatz von Japanern, die dem klassischen Konzept „Mann als Arbeiter und Ernährer, Frau als Hausfrau und Mutter" zustimmen, von 43,1\% im März 1987 auf 25\% im Jahr 2002 gesunken; dabei lehnen vor allem jüngere Frauen das bekannte Konzept ab (vgl. MOFA 2002). Es wird also deutlich, dass immer mehr Japanerinnen arbeiten wollen und sich in der einstigen Männerdomäne zu behaupten versuchen.

Diese emanzipatorische Entwicklung war auch im Videospiel durch das Erscheinen der starken, ambivalenten Frau ersichtlich; doch genauso wie sich auch heute noch Japanerinnen dafür entscheiden können, „nur“ Ehefrau und Mutter zu sein, ist auch im Videospiel das klassische Bild weiblicher Charaktere nicht verloren gegangen. Im Gegenteil lässt die Videospielindustrie gerade in den letzten Jahren immer wieder genau dieses Bild zu Vermarktungszwecken aufleben, wie zum Beispiel im Falle des Nintendo DS (Handheld aus dem Hause Nintendo, 2004) deutlich wird. Dieser nämlich setzt auf Spiele, die geradezu auf Mädchen und Hausfrauen zugeschnitten scheinen. So wundert es nicht, dass neben Super Princess Peach vor allem Titel wie Cooking Mama (2006) oder Nintendogs, bei denen es darum geht, kulinarische Köstlichkeiten auf den Tisch zu bringen oder seine eigenen Hundewelpen zu erziehen, für den Handheld erschienen sind. Sie dienen sozusagen als „Training“ für die japanische Hausfrau oder als Anreiz für junge Mädchen, in verschiedene Rollen - von der einfachen Hundehalterin bis hin zur pinken Prinzessin - schlüpfen zu können. Und schließlich lassen die bunten, schrillen Werbespots mit ihren Anime-Figuren im kawaisa-Stil ${ }^{19}$ jeg-

${ }^{19}$ Siehe beispielsweise den japanischen Werbespot zu Cooking Mama auf http://www.youtube.com/ watch? $v=$ FkaRzgfyDrg. 
lichen Zweifel daran verschwinden, dass es sich bei solchen Spielen um klares Zielgruppen-Marketing handelt.

Das Videospiel als modernes Medium japanischer Populärkultur schafft es also, verschiedene Lebensweisen widerzuspiegeln und zeigt den Strukturwandel der Gesellschaft mit unterschiedlichen Vorstellungen zum Geschlechterbild auf. Da das Videospiel ein interaktives Medium ist, ist das Ansprechen verschiedener Zielgruppen und die Erfassung möglichst vieler (teils auch konträrer) Vorstellungen ein essentieller Faktor für Vermarktung und Verkauf durch mögliche Identifikation der Spieler mit dem Dargestellten. Daraus erklärt sich auch, dass gewisse Attribute, die auf neue Geschlechterbilder schließen lassen, in den virtuellen Figuren nicht vollständig zu finden sind: Das Videospiel als populärkulturelles Produkt versucht in erster Linie zu unterhalten und nicht zu instruieren. Es soll nicht unbedingt Kritik an vorhandenen Rollenvorstellungen ausgeübt werden; vielmehr sollen die Wünsche und Vorstellungen der Zielgruppe(n) berücksichtigt werden (vgl. Sugihara/Katsurada 2002). Solche Charaktere, die sich nicht einmal ansatzweise in gewünschte Rollenbilder einordnen lassen können, erfüllen diesen Zweck nicht und sind somit für den Erfolg eines Videospiels eher hinderlich. ${ }^{20}$

Es bleibt abzuwarten, ob sich das Verhalten der Videospielindustrie in den kommenden Jahren ändern wird. Da sich aber auch ambivalente Bi-GenderCharaktere wie "Lin“ in den Videospielen des letzten Jahrzehnts stark durchgesetzt haben und es eben solche Charaktere sind, die - wie „Mitsuru“ - auch bereits Ansätze neuer Geschlechterbilder in sich tragen, erscheint mir eine optimistische Zukunftsprognose wahrscheinlich. Vielleicht wird man mit dem Medium Videospiel dann nicht mehr hauptsächlich die Koexistenz verschiedener Gender-Auffassungen, sondern auch Innovationen im Bereich der Geschlechterrollen in Verbindung bringen können.

${ }^{20}$ Hierbei muss berücksichtigt werden, dass diese Arbeit vor allem solche Videospiele untersucht hat, die den großen Videospiel-Konzernen Nintendo und Sony zugehörig sind und außerdem in Japan (und weltweit) zu großer Popularität gekommen sind. Weitere Untersuchungen zu eher unbekannteren Videospielen oder solchen Spielen kleinerer Firmen führen möglicherweise zu anderen Resultaten. 


\section{Marvin Udzik}

\section{Literatur}

Forschungsinstitut für Mittelalter und Renaissance (1985): Das Ritterbild in Mittelalter und Renaissance. Düsseldorf: Droste.

Sugihara, Yoko und Katsurada, Emiko (2002): „Gender Role development in Japanese culture: Diminishing gender role differences in a contemporary society. “In: Sex Roles: A Journal of Research (November 2002). (Via http://findarticles.com/p/articles/mi_ m2294/is_2002_Nov/ai_97728458; [Stand: 15.4.2009]).

\section{Videospiele}

Lufia, SNES, PAL-Version, Neverland/Nintendo 1996

Final Fantasy VI, Sony Playstation, PAL-Version, Square 2002

Donkey Kong, Game Boy, Nintendo 1994

Super Mario Land, Game Boy, Nintendo 1990

Super Mario RPG: Legend of the Seven Stars, SNES, NTSC-Version, Square/Nintendo 1996 Super Princess Peach, Nintendo DS, TOSE/Nintendo 2006

The Legend of Zelda: Ocarina of Time, Nintendo 64, PAL-Version, Nintendo 1998

The Legend of Zelda: The Wind Waker, Nintendo Gamecube, PAL-Version, Nintendo 2003 Grandia II, Sony Playstation 2, PAL-Version, Game Arts/Ubisoft 2002

.hack//infection, Sony Playstation 2, PAL-Version, Bandai 2004

Breath of Fire: Dragon Quarter, Sony Playstation 2, PAL-Version, Capcom 2003

Shin Megami Tensei: Persona 3, Sony Playstation 2, PAL-Version, Atlus 2008

\section{Internetquellen}

Dragon Tear (o.J.): http://www.dragon-tear.net/bofdq-englishscript.txt [Stand: 5.5.2011].

FORFEIT IsLAND (2011): „Lufia II: Rise of the Sinistrals“. http://forfeit.electromaz.com/lufia2/ script/ [Stand: 4.5.2011].

GAme FAQs (2005): „Grandia 2 Text Script“. http://www.gamefaqs.com/console/ps2/file/ 530934/34789 [Stand: 4.5.2011].

GAme FAQs (2007): „Persona 3 Script“. http://www.gamefaqs.com/console/ps2/file/932312/ 50852 [Stand: 5.5.2011].

JAPAN GAMECHARTS (2010): „Japan Gamecharts“. http://www.japan-gamecharts.com [Stand: $5.5 .2011]$.

RANSOM-WiLEY, James (2006): „Mario \& Peach can't shake gender stereotypes.“ http: //www.joystiq.com/2006/11/16/mario-and-peach-cant-shake-gender-stereotypes/ [Stand: $4.5 .2011]$.

Wikipedia (2011): „Princess Peach“. http://en.wikipedia.org/wiki/Princess_Peach

[Stand: $5 \cdot 5 \cdot 2011]$.

WiKIPEDIA (2011): „nintendogs“. http://ja.wikipedia.org/wiki/Nintendogs [Stand: 5.5.2011]. 